\title{
Mathematical models to estimate leaf area in plants of wheat
}

\author{
P. Sastre-Vázquez ${ }^{1}$, Y. Villacampa ${ }^{2}$, J. A. Reyes ${ }^{2}$, \\ F. García-Alonso ${ }^{2} \&$ F. Verdu ${ }^{2}$ \\ ${ }^{1}$ Área de Matemáticas, \\ Facultad de Agronomía de Azul-UNCPBA, Argentina \\ ${ }^{2}$ Dpto. Matemática Aplicada, Universidad de Alicante, Spain
}

\begin{abstract}
Foliar area and light penetration are two variables that have a major impact on production. The former gives an idea of the potential photosynthetic capacity of the plant, while the latter indicates the amount of light received by its different strata. In 1952, Watson introduced the Leaf Area Index (LAI) concept, which is defined as the sum of the surface area of photosynthetically active tissues (leaves, stems) present per land surface unit. On this basis, numerous researchers have underlined the close relationship between the LAI and crop yield. Great efforts have also been made to try and simplify the measurement of this parameter. The methods used up to now to obtain leaf area data involve: planimetry and the use of photoplanimeters. However, both methods have their drawbacks. The former, as well as being destructive, requires significant instrumentation, staff and time. The latter, given the high cost of photoplanimeters, is not always affordable by researchers. The aim of this study was to find a non-destructive, affordable methodology for estimating the leaf area of wheat plants. To this end, we gathered leaves of different sizes from wheat plants at different stages of growth and measured their length, maximum width and surface area. Regression curves were obtained by using length and width as independent variables, and leaf area depended on this. The equations obtained allowed us to construct a rule whereby measurement of leaf length was replaced by the surface area of the leaf being calculated. This rule allows us to estimate leaf area with sufficient precision, with an acceptable improvement in working speed and without destroying the material being measured. The models obtained are valid but in some cases can only be applied for leaves measuring more than $7 \mathrm{~cm}$ in length. For this reason, we have also obtained families of nonlinear models that are valid for all sizes of leaf.
\end{abstract}

Keywords: wheat, mathematical model, leaf area. 


\section{Introduction}

Foliar area and light penetration are two variables that have a major impact on production. The former gives an idea of the potential photosynthetic capacity of the plant, while the latter indicates the amount of light received by its different strata.

The "Leaf Area Index" (LAI) concept is defined as the total surface area of phosynthetically active tissues (leaves, ligulae, stems) present per land surface area unit. On this basis, numerous researchers have underlined the close relationship between LAI and crop yield. Great efforts have also been made to try and simplify measurement of this parameter (Francis et al. [1]; Johnson [2]; Robinson and Massengale [3]; Wendt [4]).

The methods used up to now to obtain leaf area data involve: planimetry and the use of photoplanimeters. Both methods have their drawbacks. The former, as well as being destructive, requires significant instrumentation, staff and time. The latter, given the high cost of the measurement instrument, is not always affordable by researchers.

Williams [5] developed a non-destructive method for estimating the leaf area of tomato plants, based on photographic standards of leaves at different stages of development. The standards cover all possible leaf ranges and the approximate area of each leaf is determined by their similarity.

Stickler et al. [6] found a close relationship between the product of the maximum length and maximum width of leaves of grain sorghum and leaf area. They established a relation constant of 0.747 . This relation is similar to that obtained by several authors for maize.

Makee [7] studied the relationships between foliar area of maize hybrids and the product of the maximum length and width, as well as the relationship between foliar area and each of these two measurements independently.

Chaud and Sharman [8] described three models for estimating the foliar area of maize. They found that the most accurate model was the one where foliar area was estimated by multiplying maximum length by maximum width, multiplied by a constant of 0.7370 .

Trehan et al. [9] found that the foliar area of sunflower plants could be estimated by the product of maximum leaf length and maximum width and a constant of 0.6798 .

Schneiter [10] studied the application of a formula that used the length and width of sunflower leaves, for different genotypes and densities.

From the above-mentioned studies we can deduce that the product of leaf length and width is the best way of estimating foliar area. However, either of these two measurements on their own can be used as a good estimator.

This study attempts to find a single linear measurement that is related to the surface area of the leaf, so that it allows us to create a scale where the measurements of the estimator parameter are replaced by the estimated surface area of the leaf.

This means that this study has a dual objective. Firstly, to find a nondestructive, economical methodology that allows us to estimate the foliar area of 
wheat plants, using only length or width as the explicative variable. Secondly, to find non-linear models those describe foliar area as a function of leaf length and width without having to identify a relation constant in this case. Both methodologies will be compared in future studies on the basis of new experimental data.

\section{Materials and methods}

The material used in this study was collected from the UNCPBA Faculty of Agronomy Experimental Farm in the Blue Zone - Ruta Nacional No 3, km 304.5. The soil is typical dominant ARGIUDOL, with an approximate organic material content of $6 \%$.

The climate conditions of the year were favourable for the crop, with experimental field yields ranging from $3000 \mathrm{~kg}$ and $3500 \mathrm{~kg}$ per hectare.

180 leaves of different sizes belonging to different stages of growth were collected. Real-size plotting paper replicas of the leaves were made and were used to estimate the maximum width and length of each leaf. The corresponding surface areas were weighed on an analytical balance with an accuracy of $\pm 0.1 \mathrm{mg}$, transforming the weight obtained to the corresponding surface area by using the known surface area of a $1 \mathrm{~cm}^{2}$ square of onionskin paper.

\section{Models}

\subsection{Linear models}

The problem of simple linear regression between two variables $X$ and $Y$ involves calculating the regression curve that best represents their joint distribution. This study compares the following models:

Model I: $y=\alpha_{o}+\alpha_{1} x+\varepsilon$, where: $y=$ surface area of the leaf; $x=$ maximum width of the leaf; $\varepsilon=$ random error.

Model II: $y=\beta_{o}+\beta_{1} x+\varepsilon$, where: $y=$ surface area of the leaf; $x=$ length of the leaf; $\varepsilon=$ random error.

Model I: $y=\alpha_{o}+\alpha_{1} x+\varepsilon$, where $y=$ surface area of the leaf and $x=$ maximum width has a highly significant fit and $\mathrm{R}^{2}=0.64$, with a Coefficient of Variation of 18.37. Its regression equation is:

$$
y=24.307880 x-7.038314 .
$$

Analysis of residuals was also carried out, offering no evidence of the possible presence of "outliers" or of non-compliance with the requisites of normality and homoscedasticity required for regression analysis.

Model II: $y=\beta_{o}+\beta_{1} x+\varepsilon$, where $y=$ surface of the leaf and $x=$ length of the leaf, also had a highly significant fit and $\mathrm{R}^{2}=0,82$ and a Coefficient of Variation $=12.74$. Its regression equation is:

$$
y=1.517379 x-10,620951 .
$$


Analysis of residuals showed no sign of anomalies in this case either.

Due to the fact that Model II explains $82 \%$ of the variation and its Coefficient of Variation is less than that of Model I, we concluded that wheat leaf length is the best estimator parameter of the two analysed.

For reference purposes, table 1 shows some values for total leaf length and their corresponding estimated surface area values.

Table 1: $\quad$ Estimated foliar area as a function of length.

\begin{tabular}{|c|c|}
\hline Length (cm) & Estimated area (cm) \\
\hline 10 & 4.55 \\
\hline 12 & 7.59 \\
\hline 14 & 10.62 \\
\hline 16 & 13.66 \\
\hline 18 & 16.69 \\
\hline 20 & 19.73 \\
\hline 22 & 22.76 \\
\hline 24 & 25.80 \\
\hline 26 & 28.83 \\
\hline 28 & 31.86 \\
\hline 30 & 34.90 \\
\hline 32 & 37.93 \\
\hline
\end{tabular}

In addition, although good, determining the linear model in two variables is not valid for all leaf sizes.

The model obtained is: Area $=0.57$ length +10.59 width with $R^{2}=0.94$.

\subsection{Non linear models}

On the basis of the methodology implemented in (Verdu and Villacampa [11]) we obtained families of non-linear models, of which we selected the two models that best represent the variation in foliar area in function of length and width. Both models describe foliar area for any wheat plant.

$$
\begin{gathered}
\text { Area }=(0.21 \text { length-1.39 })^{2}+(0.17 \text { width }+2.1)^{2} \quad R^{2}=0.76 \\
\text { Area }=(0.1 \text { length }+0.5)^{3}+1.5 \text { width }+2.27 ; R^{2}=0.8
\end{gathered}
$$

\section{Conclusions}

In order to obtain the foliar area from a single variable, we need to take into account the fact that the model proposed $y=1.517379 x-10.620951$ is only useful for leaves no shorter than $7 \mathrm{~cm}$. 
The experiment should be repeated to obtain more conclusive results. This is due to the following:

- The need to make use of more diverse material, as several factors affect foliar area, the most important being genetic material and climate conditions. However, we should also take into account edaphic factors (we do not take into account others, such as sowing density, as the work published shows that they do not have a significant influence).

- The importance of analysing whether the length-width ratio is maintained throughout the cycle.

The non-linear models can be used for any size of plant. This means that we have a new tool for estimating foliar area, which will be validated with new experimental data.

New sampling should thus be carried out, and it should be based not only on the crop, but also on cycle, crop location and phenological stages.

\section{References}

[1] Francis, C.A., Rutger and Palmer. (1976). A rapid method for plant leaf area estimation in maize. Crop science, Vol 9:537-539.

[2] Johnson, R.E. (1967). Comparison of methods for estimating cotton leaf area. Agronomy Journal V 59, pp:493-494.

[3] Robinson, C.D., Massengale, R.A. (1967). Use of area-Weight relationship to estimate leaf area in alfalfa. Crop Science, V 7, pp:394395.

[4] Wendt, C.V. (1967). Use of relation ship between leaf length and leaf area to estimate the leaf area of cotton, castors and sorghum. Agronomy Journal, V, 59, pp: 484-485.

[5] Williams, R.F. (1953) Estimation of leaf area for agronomic and plant physiological studies. Australian Journal Agricultural research. V l, pp:235-246.

[6] Stickler, F.C., Wearden, S., Wearden, S. and Pauli, A.W. (1961). Leaf area determination in grain sorghum. Agronomy Journal. V 53, pp:187188.

[7] Makee, R.W. (1964). A coefficient for computing leaf area in hybrid corn. Agronomy Journal, V 56, pp:240-241.

[8] Chaud, P. and Sharman, N. (1976). Constant for determining leaf area index in maize. Indian Journal of Agronomy Vol 21, pp:171-173.

[9] Trehan, K.B., Chand, H. and Mehta., S.K. (1975). Measurement of leaf area in sunflower (Helianthus annuus L.). Science \& Culture, Vol 41, pp:238-239.

[10] Schneiter, A.A. (1978). No destructive leaf area estimation in sunflower. Agronomy Journal, Vol 70, pp:141-142.

[11] Verdu, F and Villacampa, Y. (2008). A computational algorithm for the multiple generation of non linear mathematical models and stability study. Advances in Engineering Software. V 39, Issue 5, pp:430-437. 